\title{
Long-Lasting Response after Pembrolizumab in a Patient with Metastatic Triple-Negative Breast Cancer
}

\author{
Paloma Peinado ${ }^{a} \quad$ Carmen Ramírez $^{\mathrm{a}} \quad$ José Angel García-Sáenz ${ }^{\mathrm{a}}$ \\ Alejandro Pascual $^{\mathrm{b}}$ Jesús Fuentes-Antrás ${ }^{\mathrm{a}} \quad$ Natalia Vidal $^{\mathrm{a}}$ \\ Mónica Antoñanzas ${ }^{a}$ Fernando Moreno ${ }^{a}$ \\ aDepartment of Medical Oncology, Hospital Clínico Universitario San Carlos, Madrid, Spain; ${ }^{\mathrm{b}}$ Department of \\ Pathology, Hospital Clínico Universitario San Carlos, Madrid, Spain
}

\section{Established Facts}

- Breast cancer is the first cause of cancer death in women. The triple-negative subtype is associated with aggressive behavior and poor prognosis.

- Chemotherapy is the main therapeutic option available for these patients, but it is usually associated with short overall survival.

- Immunotherapy is emerging as a promising treatment for some breast cancer patients, but monotherapy studies have shown a very limited activity.

\section{Novel Insights}

- We report an impressive long-lasting tumor response and long-term survival after pembrolizumab monotherapy in a patient with metastatic triple-negative breast cancer.

- Complete response maintained for a long time after immunotherapy monotherapy is exceptional in breast cancer.

- The study of these cases is very important in order to recognize potential markers and identify which patients may benefit from treatment with checkpoint inhibitors.

\section{Keywords}

Breast cancer · Triple-negative breast cancer . Immunotherapy · Checkpoint inhibitor · Survival

\section{Abstract \\ Introduction: Breast cancer is the first cause of cancer death in women. The triple-negative subtype is associated with ag- gressive behavior and poor prognosis. Chemotherapy is the main therapeutic option available for these patients, but it is usually associated with short overall survival. Case Presenta-}

tion: We report the case of a patient diagnosed with metastatic triple-negative breast cancer with an impressive longlasting tumor response and long-term survival after pembrolizumab monotherapy. Conclusion: Immunotherapy is emerging as a promising treatment for some breast cancer patients. Nevertheless, monotherapy studies have shown a very limited activity. Nowadays, there is no good predictor biomarker. Further investigations are needed to identify the subgroup of patients who can benefit from checkpoint inhibitor treatment.

(c) 2019 S. Karger AG, Basel 


\section{Introduction}

Breast cancer is the most frequent malignancy and the leading cause of cancer death in women [1]. Triple-negative breast cancer (TNBC) represents approximately 15$20 \%$ of all breast tumors and is defined by the lack of expression of estrogen receptors, progesterone receptors, and the absence of HER 2 overexpression or amplification [2]. They are frequently high-grade tumors associated with aggressive behavior and higher rates of relapse and mortality. Despite the progress in the last few decades, metastatic disease remains incurable with the currently available therapeutic options. The median overall survival in metastatic disease is below 15 months [3].

Although breast cancer has traditionally been considered an immunologically cold tumor, nowadays we have data reporting immunogenic activity especially in triplenegative and HER2-positive subtypes [4]. This has led to the development of research studies with checkpoint inhibitors (CPIs), both in monotherapy and in combination with chemotherapy. In general, single-agent treatments have shown a promising activity but are limited to a small subset of patients [5].

Here we report a case of durable response and long survival in a TNBC patient with pembrolizumab monotherapy.

\section{Case Report}

An 80-year-old Caucasian woman with no major comorbidities presented with an inflammatory right breast mass. Ultrasound-guided biopsy showed a TNBC, and PET-CT revealed axillary lymph node and bilateral lung metastases. With the diagnosis of stage IV (cT4d N2 M1) TNBC, she was enrolled into cohort B of the Keynote-086 phase II trial (NCT02447003) and started pembrolizumab (200 mg intravenously every 3 weeks) monotherapy. She achieved an early clinical response, with a progressive improvement of the breast inflammatory mass from the first infusion of pembrolizumab (Fig. 1). In the CT scan assessment following 3 cycles of the drug, she achieved a partial response in both local and distant disease. A complete response of the lung metastases and maintained partial response of the breast mass was obtained at the sixth infusion. She did not experience drug-related toxicities with the exception of grade 1 fatigue and grade 1 hypothyroidism. After 6 months of pembrolizumab therapy, local progression was observed while she maintained a complete response of the lung metastases (Fig. 2).

Pembrolizumab was then discontinued due to local disease progression. A second line with cyclophosphamide (50 mg orally every day) was started but the patient refused the alopecia-related drug with no response. Thus, she started capecitabine (1,000 mg/ $\mathrm{m}^{2}$ orally twice daily for 2 weeks every 3 weeks). After 5 cycles, she achieved a minor response of the breast mass. Considering that she maintained a complete response of the metastatic disease for 7 months, a right mastectomy and lymphadenectomy were proposed for local control of the disease. A surgical specimen showed a $10-\mathrm{cm}$ TNBC with pectoral infiltration and absence of metastatic involvement in the 10 lymph nodes resected. After surgery,
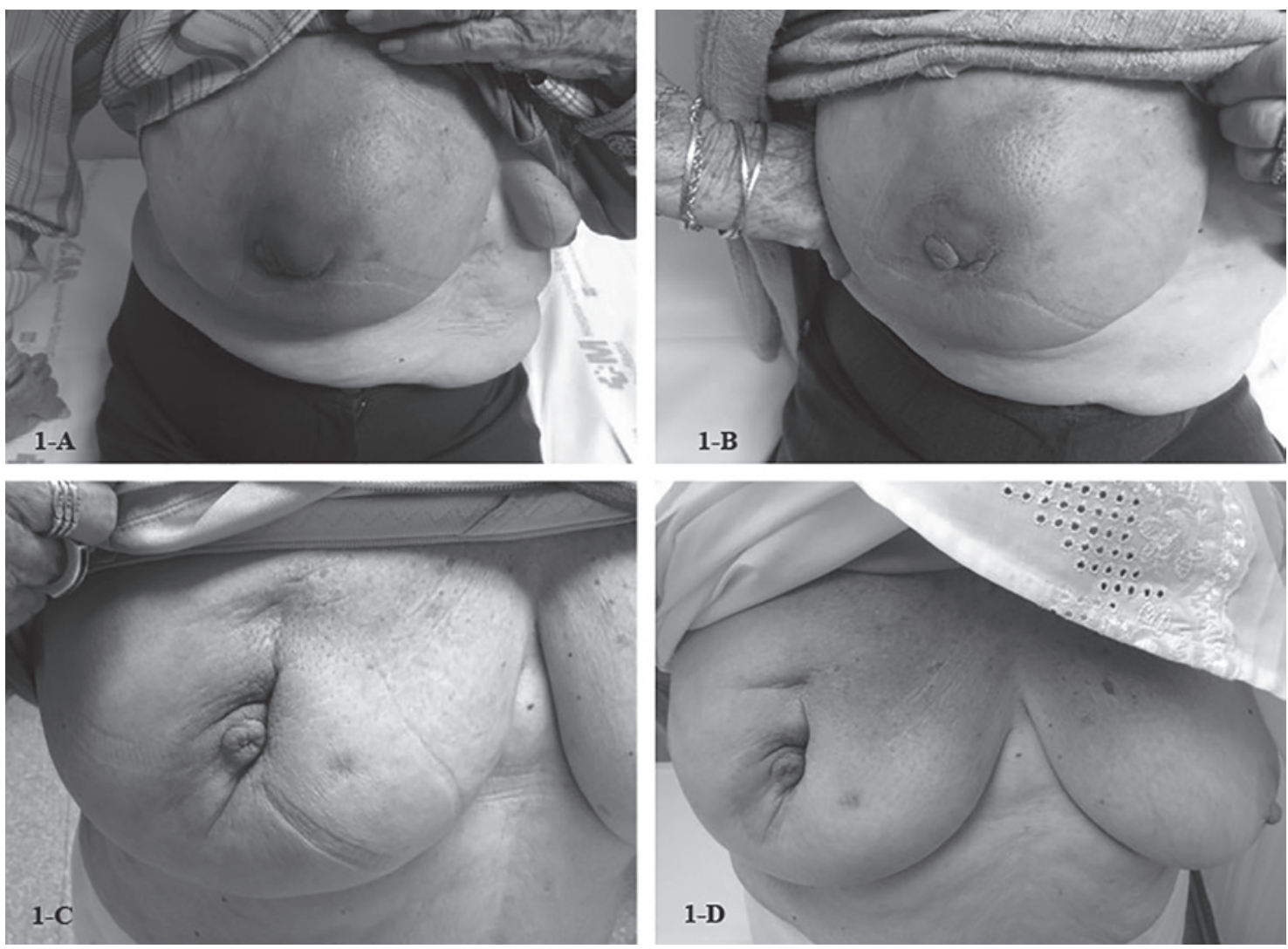

Fig. 1. Clinical response to pembrolizumab. a Baseline. b After 1 infusion. c After 3 infusions. d After 5 infusions. 
A) March 2016

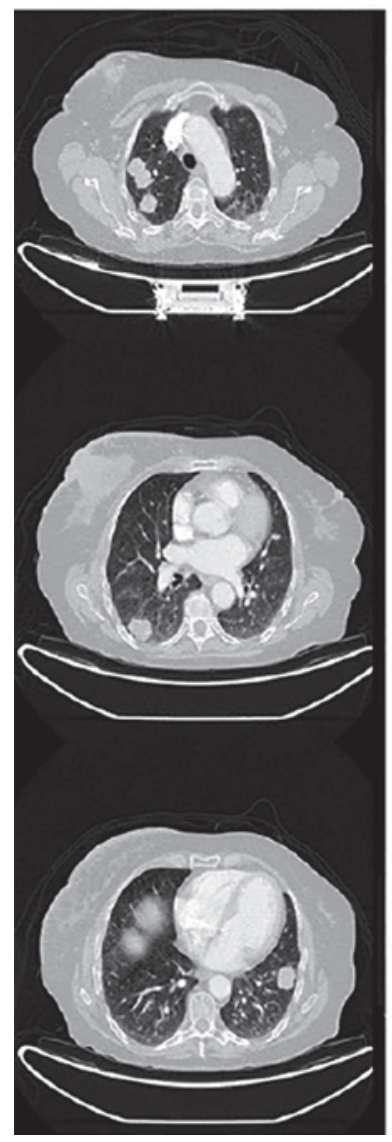

B) May 2016

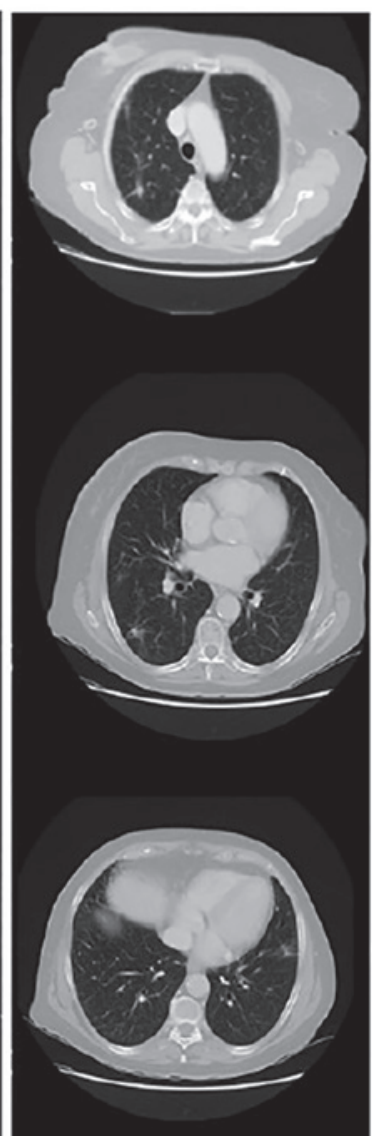

C) October 2016

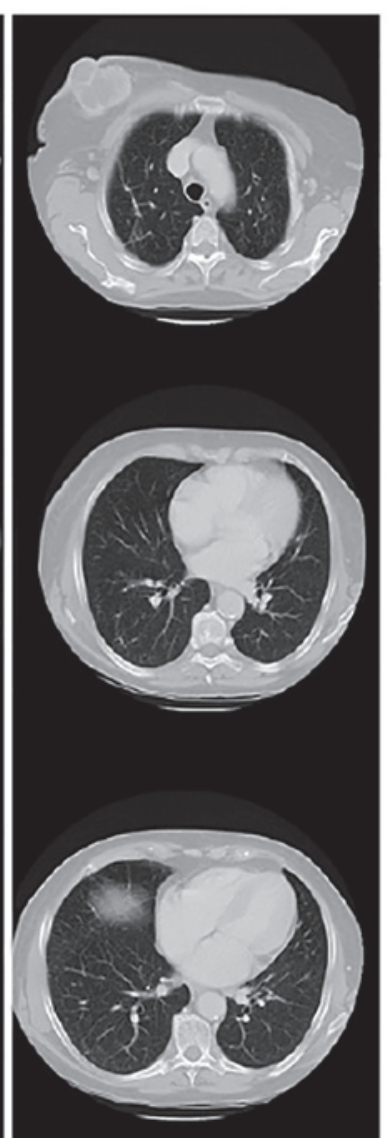

D) March 2019

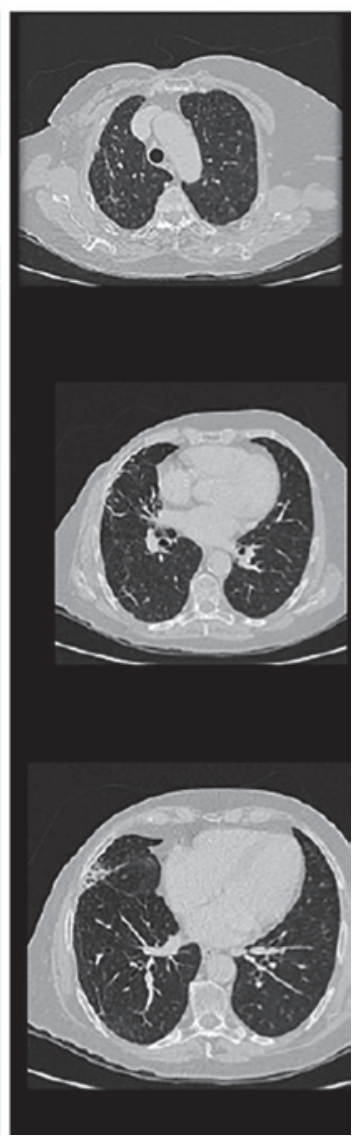

Fig. 2. Radiologic evolution. a Baseline. b Partial response after 2 months of pembrolizumab. c Complete lung response but local progression after 6 months of pembrolizumab. d Maintained complete response after breast surgery, at present.

she underwent local and regional radiotherapy (50 Gy in 25 fractions). So far, she maintains a complete tumor response following 2 years after the surgery and 3 years after the diagnosis of stage IV TNBC.

In order to study predictive factors of response, we examined both pre- and post-treatment tumor-infiltrating lymphocyte (TIL) count and programmed death ligand 1 (PD-L1) expression combined positive score (PD-L1 CPS). At baseline (first biopsy), the TIL count was $10 \%$ and PD-L1 CPS was positive (1.5). After treatment (mastectomy specimen), TIL count increased to $20 \%$ (80\% CD8+ T cells), and PD-L1 CPS became negative (0.9).

\section{Discussion}

Chemotherapy has been the only therapeutic option available for metastatic TNBC. However, it is usually associated with short-lasting responses and poor overall survival. The discovery of novel therapeutic strategies in this clinical scenario is an unmet need. In this setting, immunotherapy with CPI has emerged as a promising treatment for some breast cancer patients.
Here, we report the case of a patient with an impressive long-lasting tumor response and long-term survival of more than 3 years after an anti-programmed death- 1 (anti-PD-1) therapy. The patient was enrolled in cohort B of the Keynote-086 phase II trial, that evaluated pembrolizumab (anti-PD-1) in untreated patients with PD-L1positive metastatic TNBC [6]. Pembrolizumab showed a favorable toxicity profile with $9.5 \%$ of grade 3 toxicity. As seen in our patient, fatigue and hypothyroidism were some of the most common adverse events, 26.2 and 9.5\%, respectively. The overall response rate was $21.4 \%$ with a median duration of response of 10.4 months (4.2-19.2 months). The median progression-free survival was 2.1 months (95\% CI, 2.0-2.2) and the median overall survival 18 months (95\% CI, 12.9-23.0).

Despite the signal of activity of pembrolizumab in TNBC, predictive biomarkers are needed for selecting patients that could experience benefit with a CPI therapy.

Overall, in the metastatic setting, the response rates are higher in first-line treatments. This trend was noted in cohort A of Keynote-086 that evaluated pembrolizumab 
in 170 pretreated metastatic TNBC patients, $43.5 \%$ of whom had received at least 3 previous lines of therapy for metastatic disease [7]. The overall response rate was 5.3\%, considerably lower than in cohort B of untreated metastatic TNBC patients. Similarly, the expansion cohort of atezolizumab (anti-PD-L1) phase $1(n=115)$ showed a higher overall response rate in the first-line setting ( $26 \mathrm{vs.}$ $11 \%$ ) [8]. These findings suggest that a frontline approach results in higher responses rather than latter lines, possibly because treatment-naïve patients have greater immune competence than those who have undergone multiple lines of chemotherapy.

Stromal TIL level at baseline may also help to identify responders to immunotherapy. In the neoadjuvant setting, there is a correlation between stromal TILs and pathologic complete response $[9,10]$. In the adjuvant setting, increased stromal TIL levels at diagnosis in TNBC are associated with better outcomes after anthracyclinebased chemotherapy $[11,12]$. Stromal TILs were also examined in the Keynote-086 study, which found that median levels were higher in the treatment-naïve cohort $\mathrm{B}$ than in cohort A $(17.5$ vs. $5 \% ; p<0.001)$. When both cohorts were combined, responders had higher TIL levels than nonresponders ( 37.5 vs. $5 \%$, respectively; $p<0.001$ ) [13].

Some studies have compared the tumor microenvironment in primary tumors and their paired metastases. Overall, metastatic breast tumors have a lower number of TILs than their respective primary tumor, which indicates that immune escape plays a role in tumor progression. Stromal TIL levels may vary according to the site of metastases. Brain and liver metastases have a lower number of TILs when compared to other metastatic sites like lung and lymph nodes which may explain the limited responses seen in patients with metastases in these locations $[14,15]$.

$\mathrm{PD}-\mathrm{L} 1$ overexpression is associated with response in some studies $[8,16]$; however, other studies have shown that patients with PD-L1-negative tumors may also exhibit responses, suggesting that PD-L1 expression alone may not fully predict response to immunotherapy. This may be due to the heterogeneity of the methods used to evaluate the PD-L1 expression and the chosen cutoff values. In our case, we applied the combined score used in the Keynote-086 trial that defined positivity as a ratio of PD-L1-positive cells (tumor cells, lymphocytes, and macrophages) out of the total number of tumor cells $\geq 1 \%$ [6]. Recently, the IMpassion 130 phase 3 study showed a benefit of atezolizumab plus Nab-paclitaxel compared with Nab-paclitaxel plus placebo with a significant prolonged progression-free survival (7.2 vs. 5.5 months, HR 0.80; $p=0.002$ ). However, the improvement in progressionfree survival and overall survival was restricted to the PD-L1-positive population (PD-L1 IC of $\geq 1 \%$ by VEN-
TANA SP142 IHC assay) [17]. These results have led to the approval of atezolizumab in combination with Nabpaclitaxel for the treatment of PD-L1-positive metastatic TNBC. Nevertheless, the results of other trials with pembrolizumab in the same setting are still awaited to draw conclusions on this issue.

Interestingly, our patient had a better response in the lung metastases than in the primary tumor. In order to better understand this behavior, we performed a PD-L1 and TIL study. Although baseline biopsy showed a PD-L1 CPS that was initially positive (1.5), after pembrolizumab exposure PD-L1 CPS became negative (0.9). This could be hypothetically explained by the presence of a resistant PD-L1-negative clone that triggered the progression of residual breast tumor. Dissociated responses have been described in a minority of patients, in whom local treatments of progressing lesions should be discussed [18].

Finally, the most outstanding of this case is the longlasting response despite pembrolizumab discontinuation. This phenomenon has been previously described in other tumors like melanoma or lung cancer and it could be explained by the activation of $\mathrm{T}$ cells that stimulate a durable anti-tumor-specific immune response even after stopping the treatment $[18,19]$.

In conclusion, immunotherapy with CPIs is arising as a promising therapy in TNBC. Although response rates to single agents have been modest, some patients, like the one reported in this case, have considerable and maintained responses. Further investigations are needed to identify this subgroup of patients.

\section{Statement of Ethics}

Written informed consent was obtained from the patient for publication of this case report and accompanying images. A copy of the written consent is available for review by the Editor-in-Chief of this journal on request.

\section{Disclosure Statement}

Peinado P.: speaker fees from Kyowa Kirin, Janssen and Merck; travel support from Angelini, Merck, MSD.

Ramírez C.: speaker fees from Eisai.

García-Sáenz J.A.: consultancy/speaker fees from Novartis, Celgene, Eli Lilly, Eisai, Roche; institution research funding from AstraZeneca; travel support from Novartis, Roche, Pfizer.

Vidal N.: speaker fees from Pfizer, Kyowa Kirin, Isden, Sanofi, AstraZeneca; travel support from Merck, MSD, Pfizer, Pierre Fabre.

Antoñanzas M.: speaker fees from AstraZeneca, Eisai, Kyowa Kirin; travel support from Merck, MSD, Pierre Fabre, Osuka, Novartis.

Moreno F.: consulting or advisory role: Novartis, Pfizer, Roche, Astra Zeneca, Eisai, MSD; travel, accommodations: Roche, Pfizer; speakers bureau: Pfizer.

Pascual A. and Fuentes-Antrás J. declare no conflicts of interest. 


\section{Funding Sources}

We did not receive any specific grant from any funding agency in the public, commercial, or not-for-profit sector contributing in the planning or writing of this case report.

\section{Author Contributions}

P.P. conducted the literature review and prepared the manuscript. All authors contributed to writing the manuscript. All authors read and approved the final paper.

\section{References}

1 Bray F, Ferlay J, Soerjomataram I, Siegel RL, Torre LA, Jemal A. Global cancer statistics 2018: GLOBOCAN estimates of incidence and mortality worldwide for 36 cancers in 185 countries [Internet]. CA Cancer J Clin. 2018 Nov;68(6):394-424. [cited 2019 Mar 14] Available from: http://www.ncbi.nlm.nih. gov/pubmed/30207593

2 Denkert C, Liedtke C, Tutt A, von Minckwitz G. Molecular alterations in triple-negative breast cancer-the road to new treatment strategies [Internet]. Lancet. 2017 Jun;389(10087): 2430-42. [cited 2019 Mar 14] Available from: http://www.ncbi.nlm.nih.gov/pubmed/ 27939063

3 Gobbini E, Ezzalfani M, Dieras V, Bachelot T, Brain E, Debled M, et al. Time trends of overall survival among metastatic breast cancer patients in the real-life ESME cohort [Internet] [cited 2019 Mar 28]. Eur J Cancer. 2018 Jun;96:17-24. Available from: http://www. ncbi.nlm.nih.gov/pubmed/29660596

4 Esteva FJ, Hubbard-Lucey VM, Tang J, Pusztai L. Immunotherapy and targeted therapy combinations in metastatic breast cancer [Internet]. Lancet Oncol. 2019 Mar;20(3):e17586. Available from: https://www.sciencedir ect.com/science/article/pi i / S1470204519300269?dgcid=rss_sd_all

5 Kwa MJ, Adams S. Checkpoint inhibitors in triple-negative breast cancer (TNBC): where to go from here [Internet]. Cancer. 2018 May; 124(10):2086-103. [cited 2019 Apr 2] Available from: http://doi.wiley.com/10.1002/ cncr. 31272

6 Adams S, Loi S, Toppmeyer D, Cescon DW, De Laurentiis M, Nanda R, et al. Pembrolizumab monotherapy for previously untreated, PD-L1-positive, metastatic triple-negative breast cancer: cohort B of the phase II KEYNOTE-086 study. Ann Oncol [Internet]. 2018 Nov 26 [cited 2019 Mar 20]; Available from: http://www.ncbi.nlm.nih.gov/pubmed/ 30475947

7 Adams S, Schmid P, Rugo HS, Winer EP, Loirat $\mathrm{D}$, Awada A, et al. Pembrolizumab monotherapy for previously treated metastatic triple-negative breast cancer: cohort A of the phase II KEYNOTE-086 study. Ann Oncol [Internet]. 2018 Nov 26 [cited 2019 Mar 24]; Available from: http://www.ncbi.nlm.nih. gov/pubmed/30475950
8 Schmid P, Cruz C, Braiteh FS, Eder JP, Tolaney S, Kuter I, et al. Abstract 2986: atezolizumab in metastatic TNBC (mTNBC): longterm clinical outcomes and biomarker analyses [Internet] [cited 2019 Mar 24]. Cancer Res. 2017 Jul;77(13 Suppl):2986. Available from: http://cancerres.aacrjournals.org/lookup/doi/10.1158/1538-7445.AM2017-2986

9 Denkert C, Loibl S, Noske A, Roller M, Müller BM, Komor M, et al. Tumor-associated lymphocytes as an independent predictor of response to neoadjuvant chemotherapy in breast cancer [Internet]. J Clin Oncol. 2010 Jan;28(1):105-13. [cited 2019 Apr 1] Available from: http://www.ncbi.nlm.nih.gov/ pubmed/19917869

10 Lee HJ, Seo JY, Ahn JH, Ahn SH, Gong G. Tumor-associated lymphocytes predict response to neoadjuvant chemotherapy in breast cancer patients [Internet]. J Breast Cancer. 2013 Mar;16(1):32-9. [cited 2019 Apr 1] Available from: http://www.ncbi.nlm. nih.gov/pubmed/23593079

11 Loi S, Sirtaine N, Piette F, Salgado R, Viale G, Van Eenoo F, et al. Prognostic and predictive value of tumor-infiltrating lymphocytes in a phase III randomized adjuvant breast cancer trial in node-positive breast cancer comparing the addition of docetaxel to doxorubicin with doxorubicin-based chemotherapy: BIG 02-98 [Internet]. J Clin Oncol. 2013 Mar; 31(7):860-7. [cited 2019 Apr 1] Available from: http://ascopubs.org/doi/10.1200/JCO. 2011.41.0902

12 Adams S, Gray RJ, Demaria S, Goldstein L, Perez EA, Shulman LN, et al. Prognostic value of tumor-infiltrating lymphocytes in triplenegative breast cancers from two phase III randomized adjuvant breast cancer trials: ECOG 2197 and ECOG 1199 [Internet]. J Clin Oncol. 2014 Sep;32(27):2959-66. [cited 2019 Mar 10] Available from: www.jcowww.jco. org

13 Loi S, Adams S, Schmid P, Cortés J, Cescon DW, Winer EP, et al. LBA13Relationship between tumor infiltrating lymphocyte (TIL) levels and response to pembrolizumab (pembro) in metastatic triple-negative breast cancer (mTNBC): results from KEYNOTE-086. Ann Oncol [Internet] [cited 2019 Jul 14]. 2017 Sep 1;28(suppl_5). Available from: http://academic.oup.com/annonc/article/doi/10.1093/ annonc/mdx440.005/4109917/LBA13Relationship-between-tumor-infiltrating
14 Sobottka B, Pestalozzi B, Fink D, Moch H, Varga Z. Similar lymphocytic infiltration pattern in primary breast cancer and their corresponding distant metastases [Internet]. OncoImmunology. 2016 May;5(6):e1153208. [cited 2019 Mar 10] Available from: https:// www.tandfonline.com/doi/full/10.1080/2162 402X.2016.1153208

15 Cimino-Mathews A, Ye X, Meeker A, Argani $\mathrm{P}$, Emens LA. Metastatic triple-negative breast cancers at first relapse have fewer tumor-infiltrating lymphocytes than their matched primary breast tumors: a pilot study [Internet]. Hum Pathol. 2013 Oct;44(10): 2055-63. [cited 2019 Mar 10] Available from: https://linkinghub.elsevier.com/retrieve/pii/ S0046817713001342

16 Dirix LY, Takacs I, Jerusalem G, Nikolinakos P, Arkenau HT, Forero-Torres A, et al. Avelumab, an anti-PD-L1 antibody, in patients with locally advanced or metastatic breast cancer: a phase 1b JAVELIN Solid Tumor study [Internet] [cited 2019 Mar 24]. Breast Cancer Res Treat. 2018 Feb;167(3):671-86. Available from: http://link.springer. com/10.1007/s10549-017-4537-5

17 Schmid P, Adams S, Rugo HS, Schneeweiss A, Barrios CH, Iwata H, et al.; IMpassion130 Trial Investigators. Atezolizumab and Nab-paclitaxel in advanced triple-negative breast cancer [Internet] [cited 2019 Mar 28]. N Engl J Med. 2018 Nov;379(22):2108-21. Available from: http://www.ncbi.nlm.nih.gov/pubmed/ 30345906

18 Borcoman E, Kanjanapan Y, Champiat S, Kato S, Servois V, Kurzrock R, et al. Novel patterns of response under immunotherapy [Internet]. Ann Oncol. 2019 Mar;30(3):38596. [cited 2019 Oct 1] Available from: http:// www.ncbi.nlm.nih.gov/pubmed/30657859

19 Robert C, Ribas A, Hamid O, Daud A, Wolchok JD, Joshua AM, et al. Durable Complete Response After Discontinuation of Pembrolizumab in Patients With Metastatic Melanoma [Internet]. J Clin Oncol. 2018 Jun;36(17): 1668-74. [cited 2019 Oct 1] Available from: http://www.ncbi.nlm.nih.gov/pubmed/ 29283791 